\title{
Morphological variability of the fibularis tertius tendon in human foetuses
}

\author{
P. Karauda' ${ }^{1}$, F. Paulsen ${ }^{2,3}$, M. Polguj ${ }^{4}$, R. Diogo ${ }^{5}$, Ł. Olewnik'1(1) \\ ${ }^{1}$ Department of Anatomical Dissection and Donation, Medical University of Lodz, Poland \\ 2Institute of Functional and Clinical Anatomy, Friedrich Alexander University Erlangen-Nürnberg, Erlangen, Germany \\ ${ }^{3}$ Department of Topographic Anatomy and Operative Surgery, Sechenov University, Moscow, Russia \\ ${ }^{4}$ Department of Normal and Clinical Anatomy, Chair of Anatomy and Histology, Medical University of Lodz, Poland \\ ${ }^{5}$ Department of Anatomy, Howard University, Washington, DC, United States
}

[Received: 25 January 2021; Accepted: 11 March 2021; Early publication date: 13 April 2021]

Background: In adults, the fibularis tertius (FT) demonstrates great morphological variation. The present study classifies the types of FT insertion in human foetuses and compares their prevalence to the prevailing classification among adults. Materials and methods: Fifty spontaneously-aborted human foetuses $(19$ male, 31 female, 100 upper limbs in total) aged 18-38 weeks of gestation at death were examined. The foetuses were obtained from spontaneous abortion after parental consent. The study was performed in accordance with the legal procedures in force in Poland and with the Body Donation Programme for both adults and foetuses. Results: The most common type of FT found was type VI (32\%), characterised by a bifurcated distal attachment: a main tendon inserting onto the base of the fourth metatarsal bone, and accessory bands inserting onto the fourth interosseous space. Five other types were observed: type IV (20\%), with a single tendon inserting distally to the fascia covering the fourth interosseous space; type I (18\%), with a single tendon inserting distally onto the shaft of the fifth metatarsal bone; type $V$ $(14 \%)$, with a bifurcated arrangement comprising a main tendon characterised by a very wide insertion onto the base of the fifth metatarsal bone and an accessory band inserting onto the base of the fourth metatarsal bone; and type III (12\%) with a single tendon inserting distally onto the shaft of the fourth metatarsal bone and fascia covering the fourth interosseous space. Finally, type II (4\%) was characterised by a single tendon inserting onto the base of the fifth metatarsal bone via a very wide distal insertion.

Conclusions: The FT demonstrates high morphological variability, with the most common configuration found in adults - a single insertion onto metatarsal 5 being actually uncommonly found in foetuses. (Folia Morphol 2022; 81, 2: 451-457)

Key words: fibularis tertius, fibularis tertius tendon, anatomical variations, new classification, foetuses, variations, development

\section{INTRODUCTION}

The anterior compartment of the leg contains four muscles: the tibialis anterior, the extensor hallucis longus, extensor digitorum longus and the fibularis tertius muscle (FTM). Of this group of muscles, the FTM is the most superficial; it usually originates from

Address for correspondence: Dr. P. Karauda, Department of Normal and Clinical Anatomy, Interfaculty Chair of Anatomy and Histology, Medical University of Lodz, ul. Kościuszki 4, 90-419 Łódź, Poland, e-mail: piotr.karauda@umed.lodz.pl

This article is available in open access under Creative Common Attribution-Non-Commercial-No Derivatives 4.0 International (CC BY-NC-ND 4.0) license, allowing to download articles and share them with others as long as they credit the authors and the publisher, but without permission to change them in any way or use them commercially. 
the distal third or half of the fibula and of the intermuscular septum. The fibres of the muscle belly pass vertically downward until the muscle belly becomes the fibularis tertius tendon (FTT). After passing under the superior extensor retinaculum and inferior extensor retinaculum of the foot in the same canal as the extensor digitorum longus, the FTT inserts into the dorsal surface of the base of the fifth metatarsal bone [19]

In adults, the fibular muscle is characterised by frequent morphological variations, which mainly involve the presence of additional bands or muscles such as the fibularis digiti quinti and fibularis quartus $[5,6$, $24,25,27-29,33,37]$. The fibularis longus, fibularis brevis and fibularis tertius often demonstrate additional bands; this criterion also forms the basis of the adult classification by Olewnik et al. [24, 25, 27]. Earlier studies on foetal volatility of morphological variation in FTM are based on proximal attachment [11].

The FTM is used as a reference for the anterolateral aspect/portal during ankle arthroscopy [37]. The fibularis brevis and FTT insert at the fifth metatarsal; this implies that they can place torsional stress on areas where Jones fractures and stress fractures occur [35]. The FTM can be used in tendoplasty, tendon transfer, or resection surgeries on the foot.

The goal of our present work was to classify the types of fibularis tertius (FT) insertion in human foetuses and compare the results with those observed doe the prevailing classification among adults. It will establish the first such classification for human foetuses.

\section{MATERIALS AND METHODS}

Permission for the study was granted by the Local Bioethics Committee (agreement no RNN/130/20/KE). Fifty spontaneously-aborted human foetuses (19 male, 31 female, 100 lower limbs) aged 18-38 weeks of gestation at death were examined. The foetuses were obtained from spontaneous abortion after parental consent. The study was performed in accordance with the legal procedures in force in Poland and in accordance with the Body Donation Programme for both adults and foetuses. Their ages were determined on the basis of cranio-sacral and head measurements [26].

The leg and foot area was dissected as described previously $[21-23,25]$. The procedure began with the removal of the skin and superficial fascia of the leg up to the crural fascia. The skin and subcutaneous tissue of the foot were then removed, and then as much of the crural fascia as possible was removed without tearing the muscle bellies (starting proximal to the retinaculum). The bellies and muscle tendons were then cleaned from the medial to the lateral side. The tendon was carefully dissected to the bone attachment itself. The course of each tendon was recorded.

Upon dissection, the following features of the FT were recorded: 1) the type of FT insertion; 2) FT morphometric measurements.

Measurements were taken using an electronic digital calliper (Mitutoyo Corporation, Kawasaki-shi, Kanagawa, Japan) with an accuracy of up to $0.1 \mathrm{~mm}$. Two measurements were taken in each case and the mean values were recorded.

\section{Ethical approval and consent to participate}

The cadavers belonged to the Department of Anatomical Dissection and Donation, Medical University of Lodz.

\section{Statistical analysis}

Differences in tendon types between genders and body sides were tested using the $\chi^{2}$ test. The normality of the continuous data was tested using the Shapiro-Wilk test. As the data was not normally distributed, non-parametrical tests were used. Morphological measurements between two groups were compared using the Mann-Whitney test, and measurements between EHL types were compared using the Kruskal-Wallis test by ranks with dedicated post hoc test.

All statistical analyses were performed using Statistica 13 software (StatSoft Polska, Cracow, Poland). A p-value lower than 0.05 was considered significant, with Bonferroni correction for multiple testing. The results are presented as mean and standard deviation unless otherwise stated.

\section{RESULTS}

The fibularis tertius muscle was present in 50 cases (31 females and 19 males, $p=0.0278 ; 26$ right and 24 left limbs, $p=0.8415$ ). It occurred significantly more often in specimens with shorter lower legs $(56.2 \pm 7.2 \mathrm{~mm}$ vs. $88.0 \pm 18.9 \mathrm{~mm}, \mathrm{p}<0.0001)$ but with greater cranio-sacral length (247.2 \pm $\pm 33.05 \mathrm{~mm}$ vs. $226.76 \pm 39.90 \mathrm{~mm}, \mathrm{p}<0.0172$ ).

It was classified according to the following types, based on type of distal insertion: 
- type I - single distal attachment. The tendon inserts into the shaft of the fifth metatarsal bone (Fig. 1). This type was found in 9 cases;

- type II - single distal attachment. The tendon is characterised by a very wide insertion into the base of the fifth metatarsal bone. This type was observed in 2 cases (Fig. 1);

- type III - single distal attachment. The tendon inserts into the shaft of the fourth metatarsal bone and fascia covering the fourth interosseous space. This type was found in 6 cases (Fig. 1);

- type IV - single distal attachment. The tendon inserts to the fascia covering the fourth interosseous space. This type was found in 10 limbs (Fig. 1);

- type V - bifurcated distal attachment. The main tendon is characterised by a very wide insertion into the base of the fifth metatarsal bone, and the accessory band inserts into the base of the four metatarsal bone. This type was observed in 7 lower limbs (Fig. 1);

- type $\mathrm{VI}$ - bifurcated distal attachment. The main tendon inserts into the base of the fourth metatarsal bone, and the accessory bands inserts into the fourth interosseous space. This type was found in 16 cases (Fig. 1).

All types are also shown in the scheme (Fig. 2).

Table 1 presents the morphological parameters for the whole group and according to the above types.

Where possible, the type of insertion morphology was classified as band-shaped (18 cases in general) or fan-shaped ( 27 cases in general). The distribution of these types is presented in Table 2 .

\section{DISCUSSION}

The present study has two key values. It presents the first systematic classification of FT insertion, and highlights its variability, classifying it as band-shaped or fan-shaped in human foetuses. Earlier studies did not describe such a large morphological variability of FT insertion in human foetuses.

To understand the potential morphological variation of the FTM, it is important to explain embryological basis. The common extensor mass of the foot is connected with the peroneal mass in the early stages of development, but these diverge in a 14- $\mathrm{mm}$ embryo: the extensor mass has differentiated into the tibialis anterior muscle, extensor digitorum longus, and extensor hallucis longus. The tibialis anterior muscle demonstrates a broad tendon reaching the cuneiform primum bone. It demonstrates adult attachments in a $20-\mathrm{mm}$. embryo. The extensor digitorum longus is differentiated from the central portion of the muscle mass and is relatively more on the fibular side than in the adult. At first it ends distally in a broad flat plate which later, in a 20-mm. embryo, gives off the broad tendons to the digits. The FTM is early distinct from the extensor digitorum longus.

The FTM varies in its development and the nature of its origin and insertion. The FTM is topographically very closely related to the extensor digitorum longus, and there is every evidence that it is formed as a secondary attachment of the deep extensor stratum, which has migrated in an upward direction and has separated from the extensor digitorum brevis. In this sense, it may represent a missing tendon for the fifth toe, which became an independent muscle following migration in response to the specialised functional requirements of the human foot [14]. Therefore, like the foot to which it belongs, the FTM must be idiosyncratic feature that evolved early in human phylogenic development [11]: it is very rare in the great apes but is found in about $29.6 \%$ of gorillas (terrestrial apes). Its overwhelming presence in both humans and gorillas, and its lack or rarity in the great apes, would indicate an evolutionary acquisition related to bipedalism $[4,7-10,37]$. Hence, from an evolutionary point of view, FTM most likely serves a very important function when walking. Therefore, any morphological changes in insertion may indicate that they have not yet reached the final evolutionary stage; the FTM has been found to have a range of insertion types, and to display accessory bands [24].

Among adults, the presence or absence of the FTM depends on the population studied. The widest range in its prevalence has so far been observed in Asian populations, ranging from $38.5 \%$ to $95.5 \%[1,13$, $14,20,32]$. In North Africa, it was present in $52.8 \%$ of an Egyptian population and $67.7 \%$ of a Tunisian population [32]. In South America, it was present in $49.1 \%, 100 \%$ [30] and $93.8 \%$ of Brazilian populations [18], and interestingly, in $100 \%$ of a studied Bolivian population $[16,18,30]$. European population ranked between $38.2 \%$ and $92.9 \%[3,15,17,24,31,36]$.

In the foetus, the FTM was found to be present in only $83.16 \%$ of cases in a Polish population [11]. Interestingly, our present findings, also obtained from Polish foetuses, found it to be present in only $50 \%$.

Many FT classifications have been described $[12,14,31,34]$. However, the most recent classifica- 




Figure 1. Types of the fibularis tertius tendon: Type I of the fibularis tertius tendon. Right leg; Type II of the fibularis tertius tendon. Left leg; Type III of the fibularis tertius tendon. Right leg; Type IV of the fibularis tertius tendon. Left leg; Type V of the fibularis tertius tendon. Left leg; Type VI of the fibularis tertius tendon. Right leg; EDL — extensor digitorum longus; EDLM — extensor digitorum longus muscle; FT — fibularis tertius; FTM — fibularis tertius muscle; IIICB — third metatarsal bone; IVCB — fourth metatarsal bone; VCB — fifth metatarsal bone.

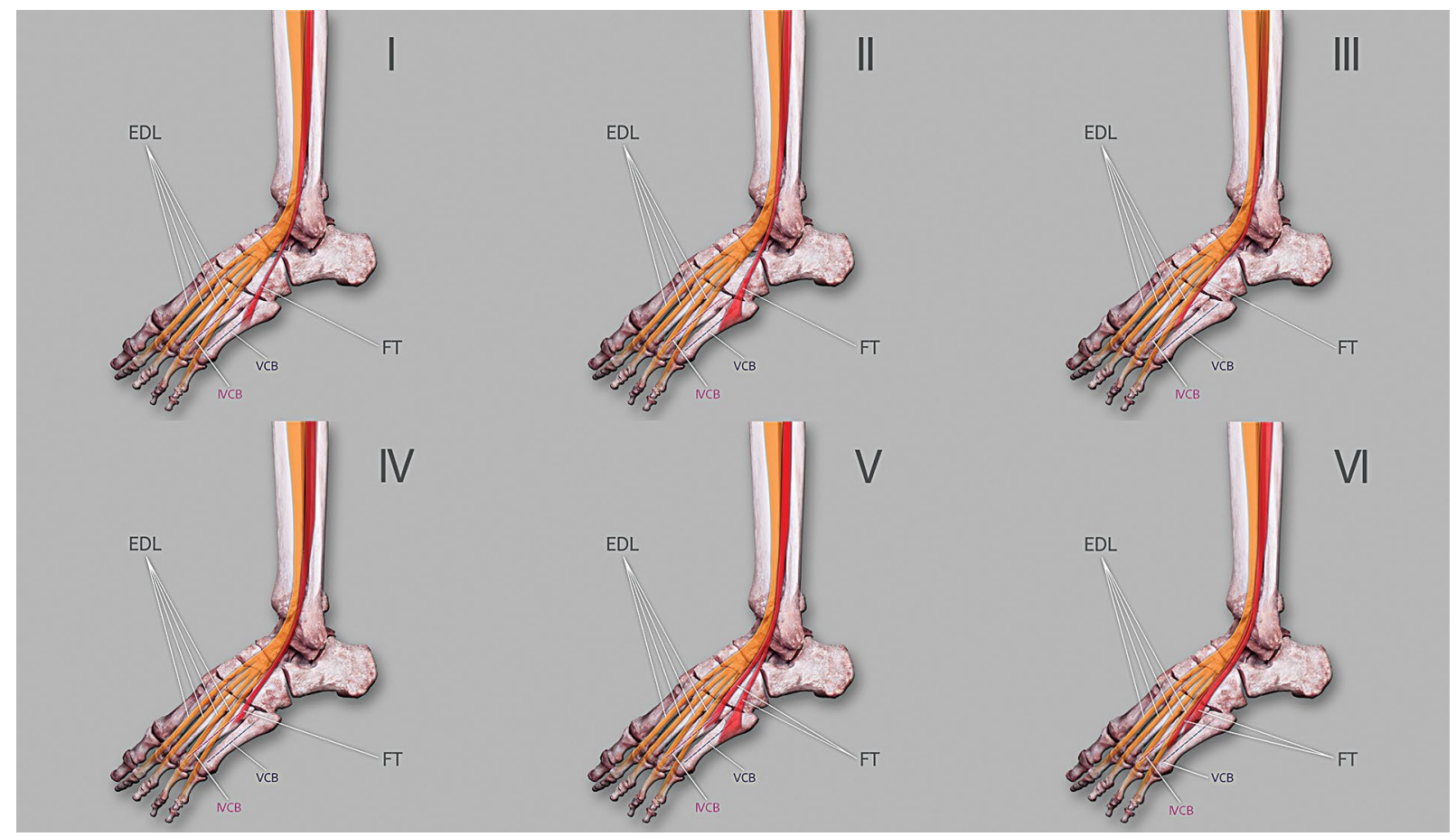

Figure 2. Scheme of all types. Left leg; EDL — extensor digitorum longus; FT — fibularis tertius; IVCB — fourth metatarsal bone; VCB — fifth metatarsal bone. 
Table 1. Morphological parameters according to type of insertion

\begin{tabular}{lcccccccc}
\hline Parameter & General & \multicolumn{9}{c}{ Insertion type } & P \\
\cline { 3 - 8 } & & $\mathbf{I}$ & II & III & IV & V & V & \\
\hline Muscle length & $12.99(4.29)$ & $13.63(2.44)$ & $11.32(1.01)$ & $10.78(0.93)$ & $11.65(0.96)$ & $15.35(4.71)$ & $14.02(6.39)$ & 0.2080 \\
Tendon width MTJ & $0.85(1.51)$ & $0.61(0.33)$ & $0.62(0.08)$ & $0.21^{*}(0.03)$ & $0.26^{*}(0.07)$ & $1.01^{*}(0.22)$ & $1.56^{*}(2.52)$ & $0.0002^{*}$ \\
Tendon thickness MTJ & $0.18(0.16)$ & $0.13(0.08)$ & $0.22(0.01)$ & $0.19(0.24)$ & $0.09(0.05)$ & $0.18(0.08)$ & $0.26(0.21)$ & 0.0163 \\
Tendon length & $11.40(3.12)$ & $11.14(2.01)$ & $12.09(1.36)$ & $11.60(0.58)$ & $13.58^{*}(1.85)$ & $9.64^{*}(3.84)$ & $10.79(4.04)$ & $0.0050^{*}$ \\
Accessory tendon length & $9.30(2.95)$ & & & & & $5.61(0.23)$ & $11.15(0.92)$ & 0.1051 \\
Distance from ExP & $3.57(1.12)$ & $2.54(0.62)$ & $3.31(1.00)$ & $4.63(0.28)$ & & $3.38(1.45)$ & $3.75(0.95)$ & 0.0179 \\
ExP width & $1.05(0.39)$ & $0.77(0.15)$ & $1.09(0.01)$ & $1.35(0.15)$ & & $1.03(0.56)$ & $1.06(0.39)$ & 0.0537 \\
ExP thickness & $0.28(0.84)$ & $0.12(0.05)$ & $0.10(0.01)$ & $0.13(0.08)$ & & $0.83(1.74)$ & $0.11(0.06)$ & 0.2262 \\
ExP width & $2.12(0.64)$ & $1.69(0.68)$ & $1.94(1.05)$ & $2.51(0.16)$ & & $2.54(0.57)$ & $1.90(0.58)$ & 0.0718 \\
ExP thickness & $0.22(0.21)$ & $0.14(0.06)$ & $0.61(0.73)$ & $0.14(0.06)$ & & $0.28(0.21)$ & $0.18(0.07)$ & 0.5020 \\
\hline
\end{tabular}

${ }^{*}$ Significant $\mathrm{P}$ according to Bonferroni correction was 0.005 ; MTJ — musculo-tendinous junction; ExP — extension point

Table 2. Shape of distal tendon insertion according to insertion type

\begin{tabular}{lcc}
\hline Insertion type & \multicolumn{2}{c}{ Insertion shape } \\
\cline { 2 - 3 } & Fan & Band \\
\hline I & $5(18.5 \%)$ & $3(16.7 \%)$ \\
II & $1(3.7 \%)$ & $0(0.0 \%)$ \\
III & $6(22.2 \%)$ & $0(0.0 \%)$ \\
IV & $0(0.0 \%)$ & $10(55.6 \%)$ \\
V & $7(25.9 \%)$ & $0(0.0 \%)$ \\
VI & $8(29.6 \%)$ & $5(27.8 \%)$ \\
\hline
\end{tabular}

tion was the 6-fold classification (I-VI) proposed by Olewnik [24]. Briefly, type I (45\%), i.e. a single distal attachment inserting into the shaft of the $V$ metatarsal bone [24], was observed in $18 \%$ of cases in the present study; type II (22\%), with a single, broad distal attachment insertion to the base of the $\mathrm{V}$ metatarsal bone [24], was present in 4\%. Type III (16.5\%), characterised by a single, very wide distal attachment to the base of the $V$ metatarsal bone, and to the base and shaft of the IV metatarsal bone, and to the fascia covering the fourth interosseous space [24], was not observed in the current study. It is possible that it appears at a later date - tendon dehiscence can occur postnatally. In addition, type IV (8.8\%), characterised by a bifurcated distal attachment into the base of the fifth metatarsal bone, and the accessory band inserts to the shaft of the $\mathrm{V}$ metatarsal bone, was not observed. Type $V(5.5 \%)$, characterised by a bifurcated distal attachment, the main tendon having a very wide insertion to the base of the $V$ meta- tarsal bone, and the accessory band inserting to the base of the IV metatarsal bone [24]. In addition, type $\mathrm{VI}$, characterised by fusion with fibularis brevis tendon, was not observed by us either.

Interestingly, of the tendon types proposed in the present study, type III, with a single distal attachment into the shaft of the fourth metatarsal bone and fascia covering the fourth interosseous space, was not observed in studies on adults, or in foetal studies [11]. Similarly, type IV, characterised by single distal attachment and tendon inserts to the fascia covering the fourth interosseous space, was not recorded by either Olewnik [24] in adults or Domagała et al. [11] in foetuses. Type V, with a bifurcated distal attachment where the main tendon has a very wide insertion into the base of the fifth metatarsal bone, and the accessory band inserts into the base of the four-metatarsal bone, was not found previously. Interestingly, type VI has not been observed in other studies, despite being the most frequent type (32\%); this was characterised by a bifurcated distal attachment where the main tendon inserts into the base of the fourth metatarsal bone, and the accessory bands inserts into the fourth interosseous space. Albay and Candan [2] also did not describe the morphological variability of fibularis tertius tendon, focusing only on the assessment of the incidence of additional fibular muscles.

Albay and Candan [2] performed morphometric measurements of FT depending on trimesters. In the second trimester, the average muscle belly length was $19.18 \mathrm{~mm}$, and the tendons were $12.13 \mathrm{~mm}$. In the third trimester, the muscle belly was $30.26 \mathrm{~mm}$, and the tendon was $15.68 \mathrm{~mm}$. Our research focused on 
morphometric measurements depending on the type of insertion. And the longest muscle belly was type $V$ $(15.35 \mathrm{~mm})$ and the shortest was type III $(10.78 \mathrm{~mm})$. The tendon length was the longest in type IV (13.59 $\mathrm{mm})$, and the smallest in type $\mathrm{V}(9.64 \mathrm{~mm})$.

A second feature in our proposed classification is insertion type. Both band-shaped and fan-shaped types were observed. Interestingly, although the presence of a band-shaped or fan-shaped type was closely correlated with the corresponding type of insertion in adults [24], no such a correlation was observed in the present study. This might be an important consideration when stripping tendons during surgery.

\section{CONCLUSIONS}

We propose a six-fold classification that can be used to elucidate the appearance of accessory tendon bands. The fibularis tertius demonstrates high morphological variability, with the most common configuration found in adults - a single insertion onto metatarsal 5 - being actually uncommonly found in foetuses.

\section{Conflict of interest: None declared}

\section{REFERENCES}

1. Adachi B. Beitrage zur anatomie der Japaner. XII. Die statistic der muskelvarietten. Z Morphol Anthr. 1909; 12: 261-312.

2. Albay S, Candan B. Evaluation of fibular muscles and prevalence of accessory fibular muscles on fetal cadavers. Surg Radiol Anat. 2017; 39(12): 1337-1341, doi: 10.1007/ s00276-017-1887-y, indexed in Pubmed: 28608130.

3. Bertelli J, Khoury Z. The peroneus tertius island muscle. Flap Surg Radiol Anat. 1991; 13(3): 243-244.

4. Boyle EK, Mahon V, Diogo R. Muscles Lost in Our Adult Primate Ancestors Still Imprint in Us: on Muscle Evolution, Development, Variations, and Pathologies. Current Molecular Biology Reports. 2020; 6(2): 32-50, doi: 10.1007/ s40610-020-00128-x.

5. Chaney ME, Dao TV, Brechtel BS, et al. The fibularis digiti quinti tendon: a cadaveric study with anthropological and clinical considerations. Foot (Edinb). 2018; 34: 45-47, doi: 10.1016/j.foot.2017.11.012, indexed in Pubmed: 29278836.

6. Demir BT, Gümüşalan $Y$, Üzel $M$, et al. The variations of peroneus digiti quinti muscle and its contribution to the extension of the fifth toe. A cadaveric study. Saudi Med J. 2015; 36(11): 1285-1289, doi: 10.15537/ smj.2015.11.12657, indexed in Pubmed: 26593160.

7. Diogo R. Introduction to evolutionary developmental pathology, or evo-devo-path: on neodarwinism, natural mutants, hopeful monsters, syndromes, genomics, variations, humans, apes, chameleons, and dinosaurs. Curr Mol Biol Rep. 2020; 6(2): 11-15, doi: 10.1007/s40610020-00133-0.
8. Diogo R, Molnar J. Comparative anatomy, evolution, and homologies of tetrapod hindlimb muscles, comparison with forelimb muscles, and deconstruction of the forelimb-hindlimb serial homology hypothesis. Anat Rec (Hoboken). 2014; 297(6): 1047-1075, doi: 10.1002/ar.22919, indexed in Pubmed: 24729440.

9. Diogo R, Molnar JL, Wood B. Bonobo anatomy reveals stasis and mosaicism in chimpanzee evolution, and supports bonobos as the most appropriate extant model for the common ancestor of chimpanzees and humans. Sci Rep. 2017; 7(1): 608, doi: 10.1038/s41598-017-00548-3, indexed in Pubmed: 28377592.

10. Diogo R, Siomava N, Gitton Y. Development of human limb muscles based on whole-mount immunostaining and the links between ontogeny and evolution. Development. 2019; 146(20), doi: 10.1242/dev.180349, indexed in Pubmed: 31575609.

11. Domagała Z, Gworys B, Kreczyńska B, et al. A contribution to the discussion concerning the variability of the third peroneal muscle: An anatomical analysis on the basis of foetal material. Folia Morphol. 2006; 65(4): 329-336, indexed in Pubmed: 17171612.

12. Ercikti N, Apaydin N, Kocabiyik N, et al. Insertional characteristics of the peroneus tertius tendon: revisiting the anatomy of an underestimated muscle. J Foot Ankle Surg. 2016; 55(4): 709-713, doi: 10.1053/j.jfas.2016.01.018, indexed in Pubmed: 26860045.

13. Jadhav Surekha $D$, et al. Fibularis tertius muscle: Cadaveric study in Indians. J Krishna Inst Med Sci Univ. 2015; 4(1): 64-69.

14. Joshi SD, Joshi SS, Athavale SA. Morphology of peroneus tertius muscle. Clin Anat. 2006; 19(7): 611-614, doi: 10.1002/ca.20243, indexed in Pubmed: 16317742.

15. Krammer EB, Lischka MF, Gruber H. Gross anatomy and evolutionary significance of the human peroneus III. Anat Embryol (Berl). 1979; 155(3): 291-302, doi: 10.1007/ BF00317642, indexed in Pubmed: 572152.

16. Larico I, Jorda L. Frecuencia del mu'sculo peroneo tertius. Rev Inv e Info Salud. 2005: 29-32.

17. Losa-Iglesias ME, Calvo-Lobo C, Rodríguez-Sanz D, et al. Fibularis tertius muscle in women and men: A surface anatomy cross-sectional study across countries. PLoS One. 2019; 14(4): e0215118, doi: 10.1371/journal. pone.0215118, indexed in Pubmed: 30964928.

18. Marin L, et al. Estudo anatomico do musculo fibular terceiro em humanos. Arq Méd ABC. 2006; 31: 23-26.

19. Moore K, Dalley A. Lower Limb. Clinically Oriented Anatomy. Lippincott Williams and Wilkins, Philadelphia 2006.

20. Nakano T. Beitrage zur Anatomie der Chinesen: Die Statistik der Muskelvarietaten. Folia Anat Jpn. 1923; 1(5): 273-282, doi: 10.2535/ofaj1922.1.5_273.

21. Olewnik $\measuredangle$, Podgórski $M$, Polguj $M$, et al. A cadaveric and sonographic study of the morphology of the tibialis anterior tendon: a proposal for a new classification. J Foot Ankle Res. 2019; 12: 9, doi: 10.1186/s13047-019-0319-0, indexed in Pubmed: 30733832.

22. Olewnik $\measuredangle$, Podgórski $M$, Polguj $M$, et al. A cadaveric study of the morphology of the extensor hallucis longus a proposal for a new classification. BMC Musculoskelet Disord. 2019; 20(1): 310, doi: 10.1186/s12891-019-2688-8, indexed in Pubmed: 31266496. 
23. Olewnik $Ł$. A proposal for a new classification for the tendon of insertion of tibialis posterior. Clin Anat. 2019; 32(4): 557-565, doi: 10.1002/ca.23350, indexed in Pubmed: 30758860.

24. Olewnik $Ł$. Fibularis tertius: anatomical study and review of the literature. Clin Anat. 2019; 32(8): 1082-1093, doi: 10.1002/ca.23449, indexed in Pubmed: 31408221.

25. Olewnik $\mathrm{t}$. Is there a relationship between the occurrence of frenular ligaments and the type of fibularis longus tendon insertion? Ann Anat. 2019; 224: 47-53, doi: 10.1016/j. aanat.2019.03.002, indexed in Pubmed: 30930196.

26. Olewnik $Ł$, Waśniewska $A$, Polguj $M$, et al. Morphological variability of the palmaris longus muscle in human fetuses. Surg Radiol Anat. 2018; 40(11): 1283-1291, doi: 10.1007/ s00276-018-2069-2, indexed in Pubmed: 30022223.

27. Olewnik $t$, Podgórski $M$, Ruzik $K$, et al. New classification of the distal attachment of the fibularis brevis: anatomical variations and potential clinical implications. Foot Ankle Surg. 2020; 26(3): 308-313, doi: 10.1016/j. fas.2019.04.002, indexed in Pubmed: 31031151.

28. Ashaolu JO, Olorunyomi OI, Opabunmi OA, et al. Surface anatomy and prevalence of fibularis tertius muscle in a south-western Nigerian population. Forensic Med Anat Res. 2013; 01(02): 25-29, doi: 10.4236/fmar.2013.12005.

29. Prakash, Narayanswamy C, Singh DK, et al. Anatomical variations of peroneal muscles: a cadaver study in an Indian population and a review of the literature. J Am Podiatr Med Assoc. 2011; 101(6): 505-508, doi: 10.7547/1010505, indexed in Pubmed: 22106198.
30. Ramirez D, Gajardo C, Caballero P, et al. Clinical evaluation of fibularis tertius muscle prevalence. Int J Morphol. 2010; 28(3), doi: 10.4067/s0717-95022010000300016.

31. Rourke K, Dafydd H, Parkin IG. Fibularis tertius: revisiting the anatomy. Clin Anat. 2007; 20(8): 946-949, doi: 10.1002/ca.20500, indexed in Pubmed: 17584875.

32. Salem A, Kader GA, Almallah A, et al. Variations of peroneus tertius muscle in five Arab populations: a clinical study. Trans Res Anat. 2018; 13: 1-6, doi: 10.1016/j.tria.2018.11.001.

33. Sookur PA, Naraghi AM, Bleakney RR, et al. Accessory muscles: anatomy, symptoms, and radiologic evaluation. Radiographics. 2008; 28(2): 481-499, doi: 10.1148/ rg.282075064, indexed in Pubmed: 18349452.

34. Stevens K, Platt A, Ellis H. A cadaveric study of the peroneus tertius muscle. Clin Anat. 1993; 6(2): 106-110, doi: 10.1002/ca.980060206.

35. Vertullo CJ, Glisson RR, Nunley JA. Torsional strains in the proximal fifth metatarsal: implications for Jones and stress fracture management. Foot Ankle Int. 2004; 25(9): 650-656, doi: 10.1177/107110070402500910, indexed in Pubmed: 15563388.

36. Witvrouw E, Borre KV, Willems TM, et al. The significance of peroneus tertius muscle in ankle injuries: a prospective study. Am J Sports Med. 2006; 34(7): 1159-1163, doi: 10.1177/0363546505286021, indexed in Pubmed: 16493171.

37. Yammine K, Erić M. The fibularis (peroneus) tertius muscle in humans: a meta-analysis of anatomical studies with clinical and evolutionary implications. Biomed Res Int. 2017; 2017: 6021707, doi: 10.1155/2017/6021707, indexed in Pubmed: 28596965. 